\title{
For which graphs does every edge belong to exactly two chordless cycles?
}

\author{
Uri N. Peled ${ }^{1}$ and Julin $\mathrm{Wu}^{2}$ \\ Dept. of Mathematics, Statistics, and Computer Science \\ (M/C 249) \\ The University of Illinois at Chicago \\ 851 S. Morgan Street \\ Chicago, IL 60607-7045
}

Submitted: December 2, 1995; Accepted: April 15, 1996.

Key Words: Chordless cycles, balanced graphs, balanced matrices

Mathematical Reviews Subject Numbers: Primary 05C75; Secondary 05C3B, 05C50, 90C35

\footnotetext{
${ }^{1}$ uripeled@uic.edu

2jwu2@uic.edu
} 


\begin{abstract}
A graph is 2-cycled if each edge is contained in exactly two of its chordless cycles. The 2-cycled graphs arise in connection with the study of balanced signing of graphs and matrices. The concept of balance of a $\{0,+1,-1\}$ matrix or a signed bipartite graph has been studied by Truemper and by Conforti et al. The concept of $\alpha$-balance is a generalization introduced by Truemper. Truemper exhibits a family $\mathcal{F}$ of planar graphs such that a graph $G$ can be signed to be $\alpha$-balanced if and only if each induced subgraph of $G$ in $\mathcal{F}$ can. We show here that the graphs in $\mathcal{F}$ are exactly the 2 -connected 2-cycled graphs.
\end{abstract}




\section{Introduction}

A graph is said to be 2-cycled if each of its edges is contained in exactly two chordless cycles. The 2-cycled graphs arise in connection with the study of balanced signing of graphs and matrices by Truemper [3] and by Conforti et al. [2], as indicated in the next three paragraphs.

A signed graph is a graph $G=(V, E)$ together with a mapping $f: E \longrightarrow$ $\{+1,-1\}$. Consider a mapping $\alpha: \mathcal{C} \longrightarrow\{0,1,2,3\}$, where $\mathcal{C}$ is the set of chordless cycles of $G$. If $\Sigma_{e \in C} f(e) \equiv \alpha(C)(\bmod 4)$ for all $C \in \mathcal{C}$, we say that the signed graph is $\alpha$-balanced. A trivial necessary condition, which we assume throughout, is that $|C| \equiv \alpha(C)(\bmod 2)$ for all $C \in \mathcal{C}$. When $\alpha=0$, this condition means that $G$ is bipartite, in which case it can be specified by its adjacency matrix $A$, and $A$ is balanced in the usual sense if and only if the signed graph consisting of $G$ and the constant mapping $f=1$ is 0-balanced. Similarly, a $\{0,+1,-1\}$-matrix $A$ specifies a signed bipartite graph, and $A$ is said to be balanced when the signed bipartite graph is 0-balanced.

It is easy to check that each graph of the following types is 2-cycled (See Figure 1):

Star-subdivision of $K_{4}$ : The result of subdividing zero or more of the three edges incident to a single vertex of $K_{4}$;

Rim-subdivision of a wheel: The result of subdividing zero or more rim edges of the wheel $W_{k}, k \geq 3$;

Subdivision of $K_{2,3}$ : The result of subdividing zero or more edges of $K_{2,3}$;

Triangles-joining: Two vertex-disjoint triangles with three vertex-disjoint paths joining them.

Note that if two nonadjacent edges of $K_{4}$ and possibly other edges are subdivided, the resulting graph is not 2-cycled. It is called a bad subdivision of $K_{4}$. Truemper [3] showed that a graph $G$ possesses a mapping $f$ that makes it $\alpha$-balanced if and only if each induced subgraph of $G$ that is a starsubdivision of $K_{4}$, a rim-subdivision of a wheel, a subdivision of $K_{2,3}$ or a triangles-joining enjoys the same property. Our main result is that these are all the 2-connected 2-cycled graphs (Clearly, a graph s 2-cycled if and only if all its 2-connected components are, so without loss of generality we may consider only 2-connected graphs): 


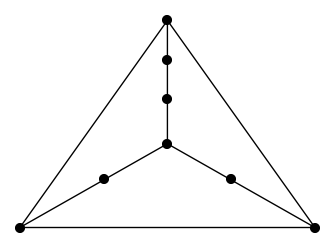

(a)

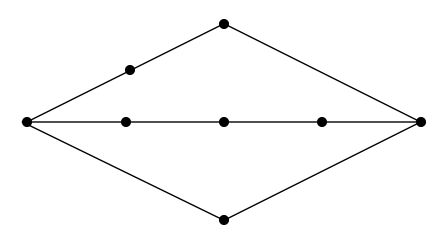

(c)

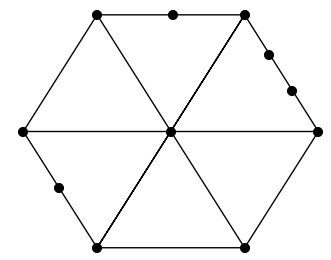

(b)

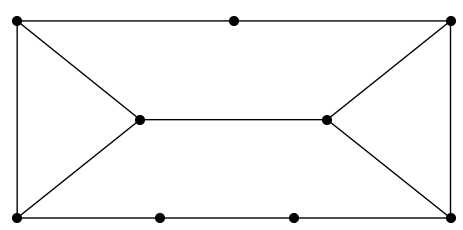

(d)

Figure 1: 2-cycled graphs. (a): Star-subdivision of $K_{4}$; (b): Rim-subdivision of a wheel; (c): Subdivision of $K_{2,3} ;(\mathrm{d})$ : Triangles-joining.

Theorem 1 (Main Theorem) A 2-connected graph is 2-cycled if and only if it is a star-subdivision of $K_{4}$, a rim-subdivision of a wheel, a subdivision of $K_{2,3}$ or a triangles-joining.

This paper is organized as follows. In Section 2 we give definitions of some new concepts. In Section 3 we define and characterize the upper and lower 2-cycled graphs; these graphs are defined so that a graph is 2-cycled if and only if it is both upper 2-cycled and lower 2-cycled. In Section 4 we study the structure of 2-cycled graphs and prove the Main Theorem. Early on (in Corollary 2) we show that the upper 2-cycled graphs are planar, and this planarity plays an important part in the proofs.

\section{Preliminaries}

We discuss only finite simple graphs and use standard terminology and notation from [1], except as indicated. We denote by $N_{G}(u)$ or simply $N(u)$ the set of vertices adjacent to a vertex $u$ in a graph $G$, and by $N_{G}(S)$ or $N(S)$ the set $\bigcup_{u \in S} N_{G}(u)$ for a vertex subset $S$. A chord of a path or a cycle is an edge joining two non-consecutive vertices of the path or cycle. A chordless 
path or cycle is one having no chord. For a path $P=\left(x_{1}, x_{2}, \ldots, x_{k}\right)$, we use the notation $P\left[x_{i}, x_{j}\right]$ for the subpath $\left(x_{i}, \ldots, x_{j}\right)$, where $1 \leq i<j \leq n$. If $e=a b$ is an edge of $G$, the contraction $G / e$ of $G$ with respect to $e$ is the graph obtained from $G$ by replacing $a$ and $b$ with a new vertex $c$ and joining $c$ to those vertices that are adjacent to $a$ or $b$. The edge set of $G / e$ may be regarded as a subset of the edge set of $G$. A minor of $G$ is a graph that can be obtained from $G$ by a sequence of vertex-deletions, edge-deletions and contractions. By subdividing an edge $e$ we mean replacing $e$ by a path $P$ joining the ends of $e$, where $P$ has length at least 2 and all of its internal vertices have degree 2. A subdivision of $G$ is a graph obtained by subdividing zero or more of the edges of $G$. The intersection (union) $G_{1} \cap G_{2}\left(G_{1} \cup G_{2}\right.$ ) of graphs $G_{1}=\left(V_{1}, E_{1}\right)$ and $G_{2}=\left(V_{2}, E_{2}\right)$ is the graph with vertex set $V_{1} \cap V_{2}$ $\left(V_{1} \cup V_{2}\right)$ and edge set $E_{1} \cap E_{2}\left(E_{1} \cup E_{2}\right)$. If $C_{1}$ and $C_{2}$ are cycles of a plane graph $G$, we say that $C_{1}$ is within (surrounds) $C_{2}$ if the area enclosed by $C_{1}$ is contained in (contains) that enclosed by $C_{2}$.

Two cycles $C$ and $C^{\prime}$ are said to be harmonic if $C \cap C^{\prime}$ is a path, as illustrated in Figure 2. If $C$ and $C^{\prime}$ are harmonic cycles of a plane graph, we can find an appropriate plane drawing of the graph such that $C^{\prime}$ is within $C$, if it is not already the case, by selecting a face within $C$ and making it the outer face.

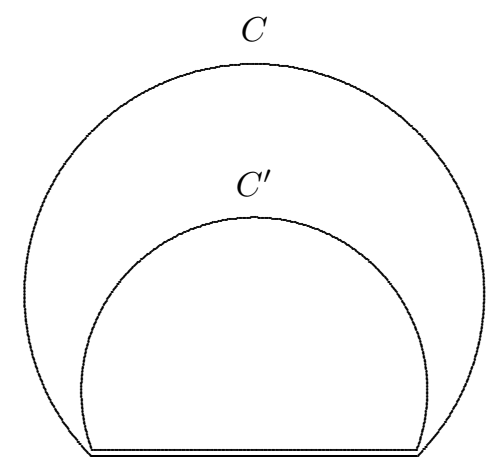

Figure 2: Harmonic cycles.

Let $C$ and $C^{\prime}$ be two cycles with a common edge $e$, and $u$ a vertex of $C^{\prime}-C$. Let $P^{\prime}$ be the maximal subpath of $C^{\prime}$ that contains $u$ and does not have internal vertices on $C$, and let $P$ be the subpath of $C$ joining the two ends of $P^{\prime}$ and containing $e$. Then $P^{\prime} \cup P$ is a cycle $C^{\prime \prime}$, as illustrated in Figure 3. The operation transforming $C^{\prime}$ into $C^{\prime \prime}$ is called grafting $C^{\prime}$ with 
respect to $C$, $e$ and $u$. An important property of this operation is that the new cycle $C^{\prime \prime}$ is harmonic with $C$. Furthermore, if the graph is a plane graph and $u$ is within $C$ (or $C^{\prime}$ surrounds $C$ ), then $C^{\prime \prime}$ is within (surrounds) $C$.

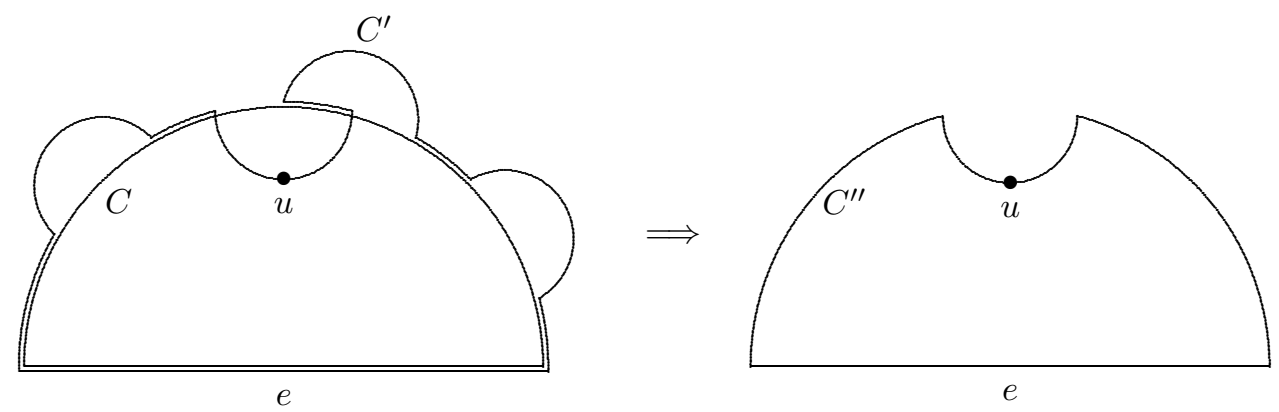

Figure 3: Grafting.

Let $P=\left(x_{1}, x_{2}, \ldots, x_{k}\right)$ be a path in $G$. If $P$ has a chord $x_{i} x_{j}$ for some $i<j-1$, we can obtain another path $P^{\prime}=\left(x_{1}, \ldots, x_{i}, x_{j}, \ldots, x_{k}\right)$ by deleting the vertices between $x_{i}$ and $x_{j}$ and adding the edge $x_{i} x_{j}$ to $P$. If $P^{\prime}$ still has chords, we can apply the same operation to $P^{\prime}$, and so on until we obtain a chordless path $P^{*}$ connecting $x_{1}$ to $x_{k}$. For a cycle $C$ of $G$ and an edge $e$ of $C$, we can apply the above operation to $C-e$ to obtain a chordless cycle $C^{*}$ containing $e$. We call the operation transforming $C$ into $C^{*}$ chord-cutting $C$ with respect to $e$. We note that if the graph is a plane graph and $C$ surrounds (is within, is harmonic with) a chordless cycle $\widehat{C}$ and $e$ is a common edge of $C$ and $\widehat{C}$, then the cycle obtained by chord-cutting $C$ with respect to $e$ again surrounds (is within, is harmonic with) $\widehat{C}$.

Let $C$ and $C^{\prime}$ be cycles of $G$, where $C$ is chordless, $e$ a common edge of $C$ and $C^{\prime}$, and $u$ a vertex of $C^{\prime}-C$. By grafting $C^{\prime}$ with respect to $C, e$ and $u$, and then chord-cutting the resulting cycle with respect to $C$ and $e$, we obtain a chordless cycle $C^{*}$. We call the operation transforming $C^{\prime}$ into $C^{*}$ harmonizing $C^{\prime}$ to $C$ with respect to $e$ and $u$. Note that the new cycle $C^{*}$ still contains $e$ and is harmonic with $C$ and chordless. Furthermore, if $G$ is a plane graph and $u$ is within $C\left(C^{\prime}\right.$ surrounds $\left.C\right)$, then $C^{*}$ is within (surrounds) $C$. After the harmonization operation we forget $C^{\prime}$ and rename $C^{*}$ as $C^{\prime}$. 


\section{Upper and lower 2-cycled graphs}

We say that a graph is upper (lower) 2-cycled if each of its edges is contained in at most (at least) two of its chordless cycles. Clearly, a graph possesses this property if and only if each 2-connected component does, but in the rest of this section we do not assume 2-connectivity. The following lemma is crucial in characterizing upper 2-cycled graphs.

Lemma 1 If $G=(V, E)$ is upper 2-cycled, so are its minors.

Proof. It suffices to show that if $G^{\prime}$ results from $G$ by deleting or contracting an edge $u v$ and $G^{\prime}$ is not upper 2-cycled, neither is $G$. Let $e=a b$ be an edge of $G^{\prime}$ that is contained in distinct chordless cycles $C_{1}^{\prime}, C_{2}^{\prime}$ and $C_{3}^{\prime}$ of $G^{\prime}$.

Case 1: $G^{\prime}=G-u v$. Note that if $u v$ is not a chord of $C_{i}^{\prime}$, then $C_{i}^{\prime}$ is also a chordless cycle of $G$; in this case, we put $C_{i}=C_{i}^{\prime}$. If $u v$ is a chord of $C_{i}^{\prime}$, then $C_{i}^{\prime} \cup u v$ is split into two chordless cycles of $G$, each consisting of $u v$ and a subpath of $C^{\prime}$ connecting $u$ to $v$; we call the one containing $e C_{i}$ and the other one $\widetilde{C}_{i}$. If $C_{1}, C_{2}$ and $C_{3}$ are distinct, then they are distinct chordless cycles of $G$ containing $e$. If they are not, we may assume $C_{1}=C_{2}$. Then $C_{1}^{\prime}$ and $C_{2}^{\prime}$ must have $u v$ as a chord, and $C_{1}, \widetilde{C}_{1}$ and $\widetilde{C}_{2}$ are distinct chordless cycles of $G$ containing $u v$.

Case 2: $G^{\prime}=G / u v$. The edge $u v$ of $G$ is contracted to a vertex $w$ of $G^{\prime}$. Because $u v \neq a b,\{a, b\} \cap\{u, v\}$ is empty or has one vertex. If it is nonempty, we assume $u=a$ without loss of generality.

If $E\left(C_{i}^{\prime}\right)$ forms a cycle of $G$, it must be a chordless cycle, and we let $C_{i}$ be that cycle. If not, $w$ must be a vertex of $C_{i}^{\prime}$, and $E\left(C_{i}^{\prime}\right)$ forms a path $P_{i}$ in $G$ connecting $u$ to $v$. Let $u_{i}^{\prime}, v_{i}^{\prime}$ be the neighbors of $u, v$ on $P_{i}$, respectively. Then $P_{i} \cup u v$ forms a cycle $C_{i}^{*}$ of $G$, and its only possible chords are $u v_{i}^{\prime}$ and $u_{i}^{\prime} v$. By chord-cutting $C_{i}^{*}$ with respect to $e$, we find a chordless cycle $C_{i}$ containing $e$.

Note that if $e$ and $u v$ are not adjacent, or if the chord $u_{i}^{\prime} v$ does not exist, then $E\left(C_{i}\right)$ is contracted to $E\left(C_{i}^{\prime}\right)$ when we contract the edge $u v$.

Now we have three chordless cycles $C_{1}, C_{2}$ and $C_{3}$ containing $e$. If they are not all distinct, say $C_{1}=C_{2}$, then $C_{1}$ is the triangle $\left\{u=a, v, b=u_{1}^{\prime}=u_{2}^{\prime}\right\}$, $C_{1}^{*}$ and $C_{2}^{*}$ both have $b v$ as a chord, and $b v$ is contained in three distinct chordless cycles of $G$, namely $\{a, v, b\}, b v \cup P_{1}^{\prime}-e, b v \cup P_{2}^{\prime}-e$.

We note that $K_{3,3}-e$ and $K_{2} \oplus 3 K_{1}$ (the graph obtained by joining every vertex of $K_{2}$ to every vertex of $3 K_{1}$ ) are not upper 2-cycled. These 
graphs are illustrated in Figure 4. Therefore we have the following corollary of Lemma 1.

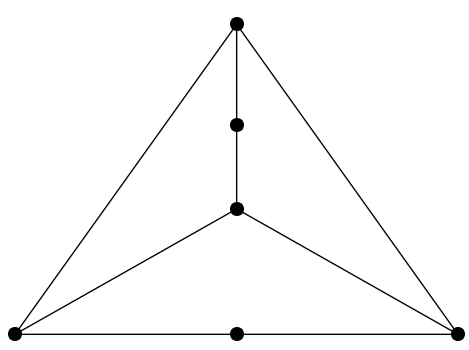

$K_{3,3}-e$

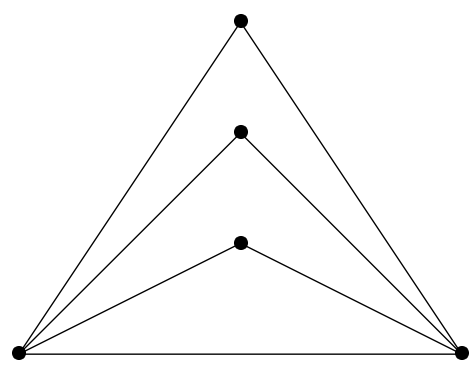

$K_{2} \oplus 3 K_{1}$

Figure 4: Forbidden minors of upper 2-cycled graphs.

Corollary 1 An upper 2-cycled graph contains no $K_{2} \oplus 3 K_{1}$ or $K_{3,3}-e$ as a minor.

Note that $K_{2} \oplus 3 K_{1}$ is a minor of $K_{5}$ and $K_{3,3}-e$ is a minor of $K_{3,3}$. By Kuratowski's Theorem, we have the following consequence of Corollary 1.

Corollary 2 An upper 2-cycled graph must be planar.

The next theorem characterizes the upper 2-cycled graphs. Although we only use its necessity part to prove the Main Theorem, it has an independent interest.

Theorem 2 A graph is upper 2-cycled if and only if it contains no $K_{2} \oplus 3 K_{1}$ or $K_{3,3}-e$ as a minor.

Proof. The necessity is Corollary 1 above. Now we prove the sufficiency. By the argument leading to Corollary 2, $G$ must be planar. Assume that, if possible, $G$ is not upper 2-cycled. We assert that $G$ has three cycles $C_{1}$, $C_{2}$ and $C_{3}$ and an edge $e$ such that the following properties hold for an appropriate plane drawing of $G$ :

1. $C_{1}, C_{2}$ and $C_{3}$ are distinct chordless cycles containing $e$;

2. $C_{2}$ is within $C_{1}$ and $C_{3}$ is within $C_{2}$; 
3. $C_{1}, C_{2}$ and $C_{3}$ are harmonic with each other.

In proving the assertion, we make use of a weaker version of Property 3, namely,

4. $C_{2}$ is harmonic with $C_{1}$ and $C_{3}$.

By the assumption that $G$ is not upper 2-cycled, it has three cycles $C_{1}, C_{2}$ and $C_{3}$ and an edge $e$ satisfying Property 1 . If two of the cycles are harmonic, we rename them as $C_{1}$ and $C_{3}$. If not, we harmonize $C_{3}$ to $C_{1}$ with respect to $e$, and the new $C_{3}$ is still different from $C_{1}$ and $C_{2}$. In any case, we may assume $C_{3}$ is within $C_{1}$. For the new $C_{1}, C_{2}$ and $C_{3}$, Property 1 still holds, but now $C_{3}$ is within and harmonic with $C_{1}$.

Next, let us consider three cases about $C_{2}$.

Case 1: $C_{2}$ has a vertex $u$ inside $C_{3}$. We harmonize $C_{2}$ to $C_{3}$ with respect to $u$ and $e$, and switch the names of $C_{3}$ and $C_{2}$. The cycles $C_{1}, C_{2}$ and $C_{3}$ now satisfy Properties 1, 2 and 4.

Case 2: $C_{2}$ has a vertex $u$ outside $C_{1}$. We select a face within $C_{3}$, make it the outer face, and switch the names of $C_{1}$ and $C_{3}$, and we are back to Case 1 .

Case 3: $C_{2}$ is between $C_{1}$ and $C_{3}$. We harmonize $C_{1}$ to $C_{2}$ and $C_{3}$ to $C_{2}$ with respect to $e$. The cycles $C_{1}, C_{2}$ and $C_{3}$ now satisfy Properties 1,2 and 4 .

Thus in all cases, Properties 1, 2 and 4 hold for $C_{1}, C_{2}, C_{3}$ and e. By planarity and Property 2 we have $C_{1} \cap C_{3} \subset C_{2}$, hence $C_{1} \cap C_{3}=\left(C_{1} \cap C_{2}\right) \cap$ $\left(C_{2} \cap C_{3}\right)$. Since each of $C_{1} \cap C_{2}$ and $C_{2} \cap C_{3}$ is a subpath of $C_{2}, C_{1} \cap C_{3}$ must be a path or the union of two disjoint paths. In the former case, $C_{1}$ is harmonic with $C_{3}$, as required. In the latter case, illustrated in Figure 5, the symmetric difference of $E\left(C_{2}\right)$ and $E\left(C_{3}\right)$ forms a cycle $C^{\prime}$, and we can find an edge $e^{\prime}$ in $C_{1} \cap C_{2}$ such that $e^{\prime}$ is also on $C^{\prime}$. Renaming $C^{\prime}$ as $C_{3}$ and $e^{\prime}$ as $e$ and chord-cutting $C_{3}$ with respect to the new edge $e$, we achieve Property 3 for the new $C_{1}, C_{2}, C_{3}$ and $e$ while Properties 1 and 2 remain valid. This completes the proof of the assertion.

It follows from the assertion that $P_{13}=C_{1} \cap C_{3}$ is a path contained in $C_{2}$ and containing $e$. Let $P_{13}^{\prime}\left(P_{31}^{\prime}\right)$ be the subpath of $C_{1}-e\left(C_{3}-e\right)$ between the ends $a$ and $b$ of $P_{13}$.

Suppose no internal vertex of $P_{31}^{\prime}$ is on $C_{2}$. Let $P_{13}^{\prime}=\left(a=x_{0}, x_{1}, \ldots, x_{k}=\right.$ $b)$, and let $i(j)$ be the largest (smallest) index such that $x_{0}, \ldots, x_{i}\left(x_{j}, \ldots, x_{k}\right)$ are on $C_{2}$, as illustrated in Figure 6 . Since $C_{1}$ and $C_{2}$ are chordless, $P_{31}^{\prime}$ and $P_{13}^{\prime}\left[x_{i}, x_{j}\right]$ are not single edges, i.e., each has an internal vertex. For the same reason, the subpath of $C_{2}$ from $x_{i}$ to $x_{j}$ that does not contain $e$ has 


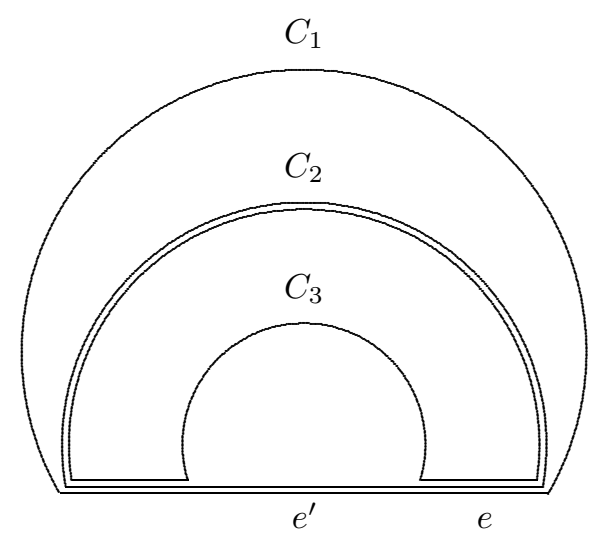

Figure 5: An illustration for the proof of the assertion.

an internal vertex. We contract $x_{0}, \ldots, x_{i}$ into one vertex and $x_{j}, \ldots, x_{k}$ into another vertex, and now $C_{1} \cup C_{2} \cup C_{3}$ is a subdivision of $K_{2} \oplus 3 K_{1}$, which has $K_{2} \oplus 3 K_{1}$ as a minor, contrary to the hypothesis. A similar argument holds if no internal vertex of $P_{13}^{\prime}$ is on $C_{2}$.

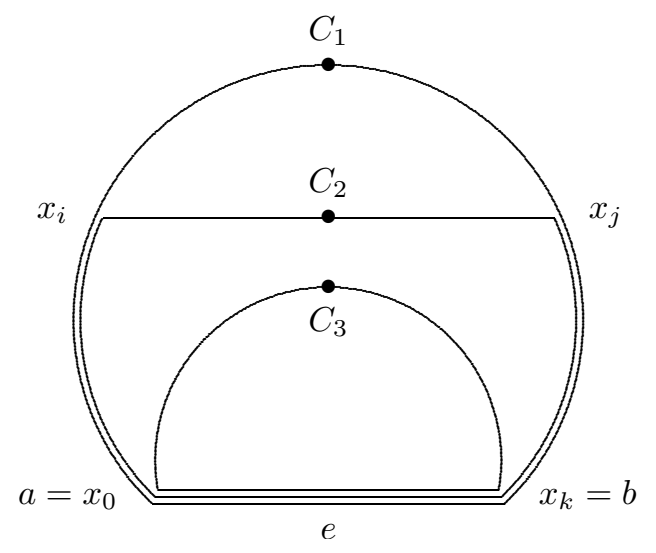

Figure 6: An illustration for the proof of Theorem 2.

If both $P_{13}^{\prime}$ and $P_{31}^{\prime}$ have an internal vertex on $C_{2}$, there is a subpath $P$ of $C_{2}$ connecting an internal vertex $d$ of $P_{13}^{\prime}$ to an internal vertex $c$ of $P_{31}^{\prime}$ such that $P$ has no internal vertex on $C_{1}$ or $C_{3}$. Without loss of generality, we assume that the cycle $C_{2}$ passes through the vertices $a, b, c, d$ in this order. Then, since $C_{2}$ is harmonic with both $C_{1}$ and $C_{3}, C_{2}$ must be $P_{13}[a, b] \cup$ 
$P_{31}^{\prime}[b, c] \cup P[c, d] \cup P_{13}^{\prime}[d, a]$, as illustrated in Figure 7. Because $C_{2}$ is a chordless cycle, each of $P_{13}^{\prime}[b, d]$ and $P_{31}^{\prime}[a, c]$ has an internal vertex. Hence $C_{1} \cup C_{2} \cup C_{3}$ is a bad subdivision of $K_{4}$, which can always be contracted to $K_{3,3}-e$, contrary to the hypothesis.

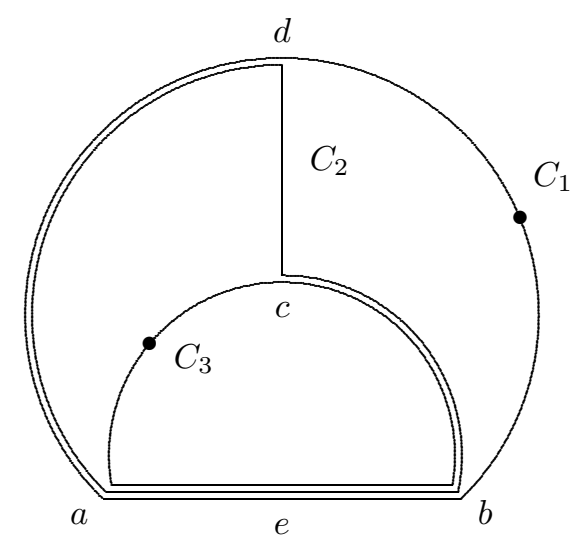

Figure 7: An illustration for the proof of Theorem 2.

The next theorem characterizes the lower 2-cycled graphs. The proof is simple and is omitted.

Theorem 3 A graph $G$ is lower 2-cycled if and only if $G$ has no bridges and every chordless cycle $C$ of $G$ satisfies at least one of the following conditions:

1. For each edge $e=u v$ of $C, G-V(C)$ has a connected component $H$ such that $N(H) \cap V(C)=\{u, v\}$;

2. $G-V(C)$ has a connected component $H$ such that $N(H)$ contains a pair of non-consecutive vertices of $C$;

3. $C$ is a triangle, and $G-V(C)$ has a connected component $H$ such that $V(C) \subseteq N(H)$.

\section{Proof of the Main Theorem}

We only need to prove the "only if" part of the Main Theorem. We do so by establishing a series of properties that a 2-connected 2-cycled graph $G$ must possess. 
THE ELECTRONIC JOURNAL OF COMBinAtorics 3 (1996), \#R14

By Corollary 2, we have the following:

Property $1 G$ is planar.

Property 2 For each edge ab of $G, G-\{a, b\}$ has at most two connected components.

Indeed, otherwise there would be three chordless cycles containing $a b$.

We call an edge $a b$ critical if $G-\{a, b\}$ has exactly two connected components.

Let $F$ be any face of a plane drawing of $G$. The boundary of $F$ is a cycle $C$ by 2-connectivity, and we call it a face-cycle. If $F$ is the outer face of a plane drawing $D$ of $G$, we call $C$ the outer cycle of $D$. Suppose the outer cycle $C$ has a critical edge $a b$. We denote by $H$ the connected component of $G-\{a, b\}$ not containing the other vertices of $C$. By 2-connectivity we have $N(H) \cap V(C)=\{a, b\}$. We can find another plane drawing $D^{\prime}$ of $G$ by moving $H$ outside of $C$. If $C^{\prime}$ denotes the new outer cycle, then $V\left(C^{\prime}\right) \supset V(C)$, and $a b$ is a chord of $C^{\prime}$, rather than an edge of it. We call the operation transforming $D$ into $D^{\prime}$ flipping. If $C^{\prime}$ still has critical edges, we repeat this operation. In a finite number of steps, we obtain a plane drawing of $G$ whose outer cycle $C^{*}$ has no critical edges. We now assert that $C^{*}$ is chordless. If not, a chord $a b$ would spilt the cycle $C^{*}$ into two cycles $C^{\prime}$ and $C^{\prime \prime}$, and we may assume that $C^{\prime}$ is chordless. There must be a vertex $u$ within $C^{\prime}$, for otherwise there would be no other chordless cycles containing an edge from $C^{\prime}-a b$. Let $H$ be the connected component of $G-V\left(C^{\prime}\right)$ containing $u$. The set $N(H) \cap V\left(C^{\prime}\right)$ cannot be the two ends of an edge from $C^{\prime}-a b$, because by Property 2 such an edge would be a critical edge on the outer cycle $C^{*}$. Thus $C^{\prime}$ does not satisfy Condition 1 of Theorem 3, and it must satisfy Condition 2 or 3 . We can therefore find a chordless path $P$ connecting two non-consecutive vertices of the path $C^{\prime}-a b$ such that all the internal vertices of $P$ are from $H$, as illustrated in Figure 8. It is easy to see that $C^{*} \cup\{a b\} \cup P$ can be contracted to $K_{2} \oplus 3 K_{1}$, contradicting Corollary 1 . This proves the assertion.

Recall that the flipping operation adds a new chord of the new outer cycle. Hence by the assertion, no flipping ever takes place, and the outer cycle $C$ of the arbitrary drawing $D$ is chordless. Since each face of a plane drawing can be drawn as the outer face, we have established the following two properties:

Property 3 G has no critical edge. 


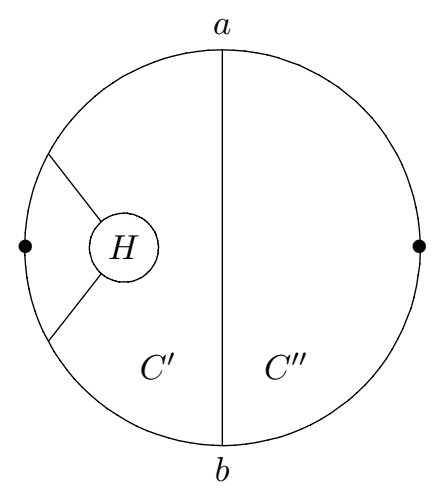

Figure 8: An illustration for the proof of the assertion.

Property 4 In each plane drawing of $G$, each face-cycle is chordless.

In each plane drawing, each edge $e$ belongs to two face-cycles by 2connectivity. The latter are chordless by Property 4, and must be the only chordless cycles containing $e$ since $G$ is 2-cycled. We therefore conclude the following:

Property 5 In each plane drawing of $G$, each chordless cycle is a face-cycle.

Another property of $G$ is given below.

Property 6 At least one of the face-cycles is not a triangle if $G \neq K_{4}$.

Suppose to the contrary that every face-cycle of $G$ is a triangle. Let $C$ be the outer cycle with vertices $a, b$ and $c$. Without loss of generality, let $k \geq 3$ be the degree of $a$, and let $a x_{1}, a x_{2}, \ldots, a x_{k}$ be the edges incident with $a$ in counterclockwise order, where $b=x_{1}$ and $c=x_{k}$. Then by our assumption, $x_{1} x_{2}, x_{2} x_{3}, \ldots, x_{k-1} x_{k}$ must be edges of $G$, as illustrated in Figure 9 .

If $k \geq 4$, there is an edge $e=x_{i} x_{j}, j-i>1, e \neq x_{1} x_{k}$, for otherwise $\left\{x_{1}, \ldots, x_{k}\right\}$ is a chordless cycle, hence a face-cycle by Property 5 , but it is not a triangle. But then the triangle $\left\{a, x_{i}, x_{j}\right\}$ is not a face-cycle, contradicting Property 5 . Therefore $k$ must be 3 , and for each $\{p, q, r\} \subseteq\left\{a, x_{1}, x_{2}, x_{3}\right\}$, the triangle $\{p, q, r\}$ is a face-cycle by Property 5 . Therefore $G$ has no other vertices, and so $G=K_{4}$.

By Property 6, we may assume that we have chosen a plane drawing of $G$ whose outer cycle $C$ has length at least 4 . We make this assumption 


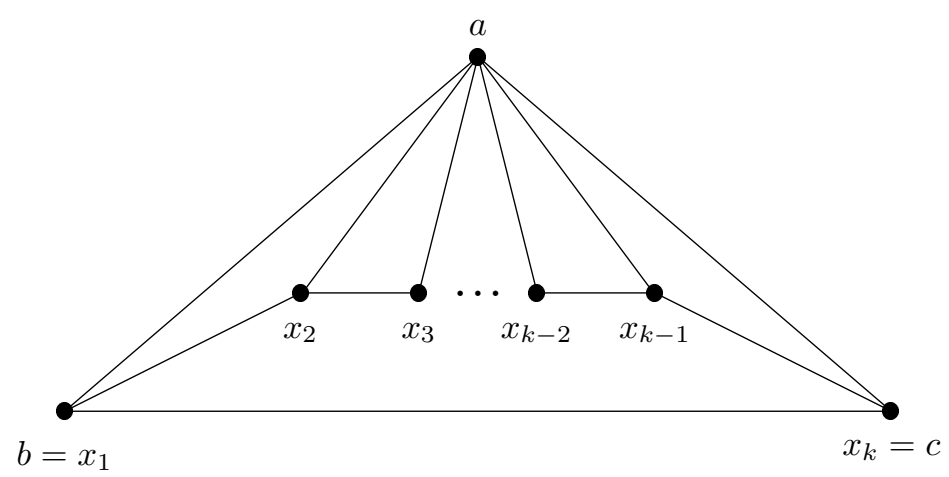

Figure 9: An illustration for the proof of Property 6.

for the rest of the proof, and use the notations $N_{C}(u)=N(u) \cap V(C)$ and $N_{C}(S)=N(S) \cap V(C)$ for a vertex $u$ and a vertex-subset $S$.

Property 7 For each connected component $H$ of $G-V(C), N_{C}(H)$ contains a pair of non-consecutive vertices along $C$.

In fact, $N_{C}(H)$ cannot be empty or a single vertex by 2-connectivity. Neither can it be the two ends of an edge $e$ of $C$, since otherwise $e$ would be a critical edge by Property 2, contrary to Property 3. Therefore $N_{C}(H)$ contains a pair of non-consecutive vertices along $C$.

Property $8 G-V(C)$ is connected.

If not, $G-V(C)$ would have at least two connected components $H_{1}$ and $H_{2}$. For $i=1,2, N_{C}\left(H_{i}\right)$ contains a pair of non-consecutive vertices $a_{i}, b_{i}$ on $C$ by Property 7 . we can find a path $P_{i}$ connecting $a_{i}$ to $b_{i}$ all of whose internal vertices are from $H_{i}$. By planarity, $P_{1}$ and $P_{2}$ do not intersect except possibly at the ends. Therefore the minor $C \cup P_{1} \cup P_{2}$ can be contracted to $K_{2} \oplus 3 K_{1}$, contrary to Corollary 1 .

Property $9 G-V(C)$ contains no cycle.

If $G-V(C)$ contains a cycle, it must contain a chordless cycle $C^{\prime}$. There exists vertex-disjoint paths $P_{1}$ and $P_{2}$ between $C$ and $C^{\prime}$ (this can be seen by adding a new vertex $s$ adjacent to every vertex of $C$ and another new vertex $t$ adjacent to every vertex of $C^{\prime}$ without destroying 2-connectivity, and then 
applying Menger's Theorem to $s$ and $t$ ). Let $x_{i}$ and $y_{i}$ be the ends of $P_{i}$ on $C$ and $C^{\prime}$, respectively.

If $x_{1}$ and $x_{2}$ are not consecutive along $C$, let $y^{\prime}$ be a third vertex on $C^{\prime}$, and let $e$ be any edge of the subpath of $C^{\prime}$ from $y_{1}$ to $y_{2}$ that avoids $y^{\prime}$, as illustrated in Figure 10. Then $e$ belongs to three chordless cycles of the minor $C \cup C^{\prime} \cup P_{1} \cup P_{2}$, contrary to Lemma 1 .

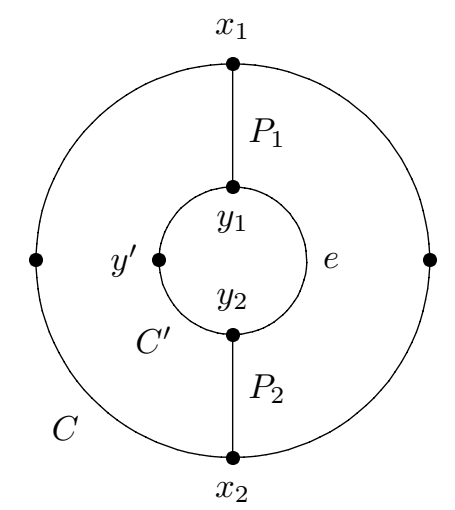

Figure 10: An illustration for the proof of Property 9.

Therefore we may assume that $x_{1}$ and $x_{2}$ are consecutive along $C$, and similarly $y_{1}$ and $y_{2}$ are consecutive along $C^{\prime}$. Then since the edge $x_{1} x_{2}$ of $C$ is not critical by Property $3, G-\left\{x_{1}, x_{2}\right\}$ has a shortest path $P_{3}$ from $C$ to $C^{\prime}$. Let $x_{3}$ and $y_{3}$ be the ends of $P_{3}$ on $C$ and $C^{\prime}$, respectively. If $P_{3}$ and $P_{1} \cup P_{2}$ are disjoint, then since $C$ has at least four vertices, we can forget $P_{1}$ or $P_{2}$ and then we are back to the previous case. Otherwise, let $z$ be the first vertex of $P_{3}$ that belongs to $P_{1} \cup P_{2}$. We may assume without loss of generality that $z$ is on $P_{1}$, as illustrated in Figure 11.

Consider the minor $M=C \cup C^{\prime} \cup P_{1} \cup P_{2} \cup P_{3}\left[x_{3}, z\right]$ of $G$. The edge $y_{1} y_{2}$ is on three chordless cycles of $M$, namely $C^{\prime}, P_{1} \cup P_{2} \cup\left\{x_{1} x_{2}, y_{1} y_{2}\right\}$, and $P_{3}\left[x_{3}, z\right] \cup P_{1}\left[z, y_{1}\right] \cup\left\{y_{1} y_{2}\right\} \cup P_{2} \cup P^{\prime}$, where $P^{\prime}$ is the subpath of $C$ from $x_{2}$ to $x_{3}$ that avoids $x_{1}$. This contradicts Lemma 1 , thereby proving Property 9 .

By Property 8 and Property $9, G-V(C)$ is a tree $T$.

Property 10 T must be a path.

If $T$ is not a path, it has a vertex $v$ such that $\operatorname{deg}_{T}(v) \geq 3$. By Property 7 , $N_{C}(T)$ has two non-consecutive vertices $a$ and $b$ along $C$. The forest $T-v$ 


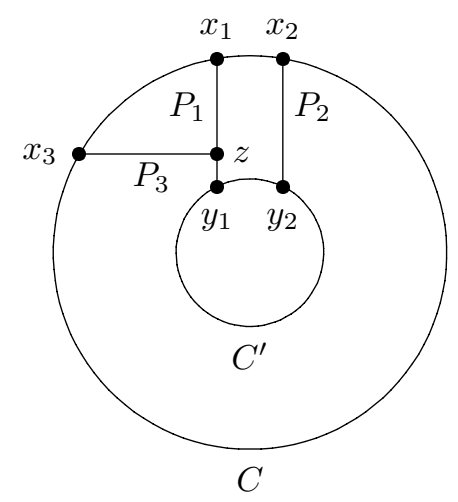

Figure 11: An illustration for the proof of Property 9.

has connected components $T_{1}$ and $T_{2}$ (possibly identical) such that $\{a\} \subseteq$ $N_{C}\left(T_{1}\right) \cup N_{C}(v)$ and $\{b\} \subseteq N_{C}\left(T_{2}\right) \cup N_{C}(v)$. Let $T_{3}$ be a connected component of $T-v$ distinct from $T_{1}$ and $T_{2}$. Since $v$ is not a cut vertex of $G$ by 2connectivity, there exists a vertex $c \in N_{C}\left(T_{3}\right)$. Let $P$ be a path from $v$ to $c$ via $T_{3}$, as illustrated in Figure 12.

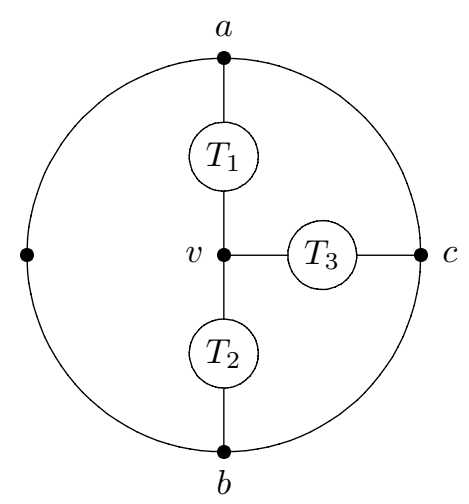

Figure 12: An illustration for the proof of Property 10.

We contract $T-T_{3}$ to a single vertex $w$, which becomes an end of $P$. Consider the minor $M=C \cup P \cup\{w a, w b\}$ of $G$. If $c \neq a, b$, then, as illustrated in Figure 13 (a), $M$ is a bad subdivision of $K_{4}$, which is not upper 2-cycled. Otherwise we may assume that $c=a$, as illustrated in Figure $13(\mathrm{~b})$, and we contract the edge $w b$ of $M$ to obtain a subdivision of $K_{2} \oplus 3 K_{1}$, which is not 
upper 2-cycled. In both cases, Lemma 1 is contradicted.

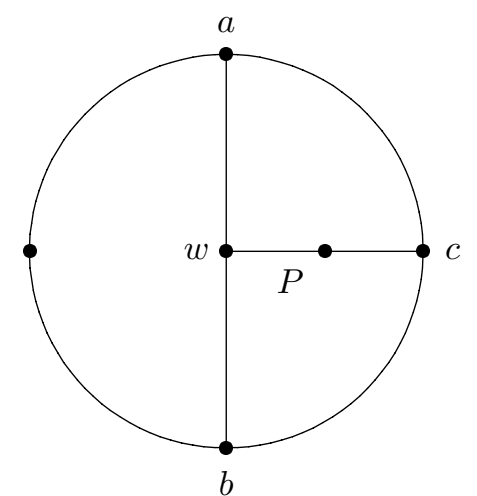

(a)

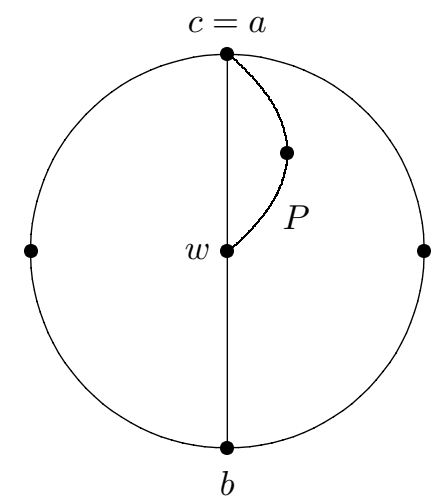

(b)

Figure 13: Illustrations for the proof of Property 10. (a): $c \neq a, b ;(\mathrm{b}): c=a$.

Property 11 If $u$ and $v$ are the two ends of the path $T$ and $u \neq v$, then $N_{C}(u)$ and $N_{C}(v)$ are nonempty, and each of $N_{C}(T-u)$ and $N_{C}(T-v)$ consists of either a single vertex or a pair of consecutive vertices along $C$.

The sets $N_{C}(u)$ and $N_{C}(v)$ (and hence also $N_{C}(T-u)$ and $N_{C}(T-$ $v)$ ) are nonempty by 2 -connectivity. Assume that $N_{C}(T-v)$ contains nonconsecutive vertices $a$ and $b$ along $C$. We contract $T-v$ to a vertex $w$, and let $P$ be a path from $w$ to $C$ via $v$. Then we argue about the minor $M=C \cup P \cup\{w a, w b\}$ as in Property 10. Similarly, $N_{C}(T-u)$ has no non-consecutive vertices along $C$.

Now we are ready to list all the possible 2-connected 2-cycled graphs, and thereby prove Theorem 1 , by considering all possibilities for $T$.

Case 1: $T$ is a single vertex $v$. Then $G$ is a rim-subdivision of a wheel $W_{k}$ with $k \geq 3$ if $\operatorname{deg}_{G}(v) \geq 3$, and by Property $7 G$ is a subdivision of $K_{2,3}$ if $\operatorname{deg}_{G}(v)=2$.

Case 2: $V(T)=\{u, v\}$. If each of $N_{C}(u)$ and $N_{C}(v)$ has only one vertex, then the two vertices are distinct and non-consecutive by Property 7 , so $G$ is a subdivision of $K_{2,3}$. If one of $N_{C}(u)$ and $N_{C}(v)$ has one vertex and the other has two (necessarily consecutive by Property 11), then $N_{C}(u) \cap N_{C}(v)=\emptyset$ by Property 7 and so $G$ is a star-subdivision of $K_{4}$. If both $N_{C}(u)$ and 
$N_{C}(v)$ (distinct by Property 7 ) have two vertices (necessarily consecutive by Property 11), then $G$ is a triangles-joining or a rim-subdivision of the wheel $W_{4}$ depending on whether $N_{C}(u) \cap N_{C}(v)$ is empty or not.

Case 3: $T$ is a path of length at least 2, with ends $u, v$. Neither $N_{C}(T-u)$ nor $N_{C}(T-v)$ contains a pair of non-consecutive vertices of $C$ by Property 11 , whereas $N_{C}(T)$ does by Property 7 . So $N_{C}(u) \cup N_{C}(v)$ must contain a pair $a, b$ of non-consecutive vertices, with $a \in N_{C}(u)$ and $b \in N_{C}(v)$. Moreover, $N_{C}(x)$ does not meet $\{a, b\}$ for any internal vertex $x$ of $T$. If $y \in N_{C}(x)$ for some internal vertex $x$ of $T$, then by the above and Property 11, $y$ must be different from and adjacent to both $a$ and $b$. For the same reason, $N_{C}(u) \subseteq\{y, a\}$ and $N_{C}(v) \subseteq\{y, b\}$, and $N_{C}(z) \subseteq\{b, y\} \cap\{a, y\}=\{y\}$ for all internal vertices $z$ of $T$. Therefore $G$ must be a rim-subdivision of a wheel with center $y$. If $N_{C}(x)$ is empty for every internal vertex $x$ of $T$, then the argument is similar to the one of Case 2.

\section{References}

[1] J.A. Bondy and U.S.R. Murty. Graph Theory with Applications. 1976. Macmillan, London, 1976.

[2] M. Conforti, G. Cornuéjols, A. Kapoor, K. Vušković, and M.R. Rao. Balanced matrices. In J.R. Birge and K.G. Murty, editors, Mathematical Programming State of the Art 1994, pages 1-33. The University of Michigan, 1994.

[3] K. Truemper. Alpha-balanced graphs and matrices and $G F(3)$-representability of matroids. Journal of Combinatorial Theory B, 32:112-139, 1982. 